\title{
Experimental characterization of mechanical properties of the cement-aggregate interface in concrete
}

\author{
M. Jebli ${ }^{\mathrm{a}, \mathrm{c}, *}$, F. Jamin ${ }^{\mathrm{a}, \mathrm{b}}$, E. Malachanne ${ }^{\mathrm{c}}$, E. Garcia-Diaz ${ }^{\mathrm{c}}$, M.S. El Youssoufi ${ }^{\mathrm{a}, \mathrm{b}}$ \\ ${ }^{a}$ LMGC, Univ. Montpellier, CNRS, Montpellier, France \\ ${ }^{\mathrm{b}}$ MIST Lab., IRSN, CNRS, Univ. Montpellier, France \\ ${ }^{\mathrm{c}} \mathrm{C} 2 \mathrm{MA}$, Ecole des mines d' Alès, Alès, France
}

A B S T R A C T

The microstructure of the Interfacial Transition Zone (ITZ) between the aggregates and the cement paste is characterized by a higher porosity than that of the bulk paste. The particular properties of this zone strongly influence the mechanical behavior of concrete. Microscopic cracks, which develop during subse-quent loading, appear either in the matrix (cement paste or mortar) or along the cement-aggregates interface. Cracks could be caused by either tensile, shear strengths or by combinations of both. In this work, the mechanical properties of the cement paste - aggregate sample are experimentally studied. The experimental tests are performed on parallelepipedic samples at classical aggregate scale (one cen-timeter sections). These samples are composed of limestone aggregates and Portland cement paste, here-after named "composite". The cement paste is prepared with a water/cement ratio of 0.5. The shape of the prepared composites makes them convenient for direct tensile and shear tests. At different stages of hydration, we performed direct tensile and shear tests on the composites by means of specific devices. The same tests were carried out on the cement paste in order to compare with the composite results. The analysis of the experimental results showed that the tensile strength of the cement-aggregate inter-face was about $30 \%$ lower than that of the cement paste tensile strength. Also, the shear strength of the cement-aggregate interface was smaller than the shear strength of the cement paste. In the same way as macroscopic Mohr-Coulomb criterion, we observed an increase of shear strength when normal stress increased. It provides access to a local cohesion (c) and a local friction angle $(\Phi)$ at classical aggregate scale.

Keywords:

Concrete

Cement-aggregate interface

Direct tensile tests

Direct shear tests

Tensile strength

Shear strength

Experimental characterization

\section{Introduction}

The cement-aggregate interface is one of the key factors associated to the resistance and durability of concrete. To understand this complex material, characterization of the properties of the cement-aggregate interface is an essential element needed to model the intrinsic properties of the three phases (aggregates, cement paste and interface). While the existence and formation

\footnotetext{
* Corresponding author at: LMGC, Univ. Montpellier, CNRS, Montpellier, France. E-mail address: mouad.jebli@umontpellier.fr (M. Jebli).
}

of the cement-aggregate interface are now well admitted in literature, the mechanical properties of this zone are unknown and remain a rarely treated subject.

The cement-aggregate interface is considered an additional phase introduced by the presence of aggregates. The bond is a result of mechanical interlocking provided by epitaxic growth of cement hydration products on the aggregate surface and by chemical reactions between the cement paste and aggregate [20].

The effect of the bond on concrete performance is a controversial subject, however there seems to be a relation between the strength of concrete and the strength of the bond [2]. In most of 
the investigations in literature, the parameters used for characterization of the interface are empirical. The experimental data parameters are not always consistent and show a wide variability.

It is generally admitted by most researchers that the microstructure of the interface zone between the aggregates and the cement paste is characterized by a higher porosity. [26] formalized this concept of cement-aggregate interface. This work was pursued by Mindess et al. [16] and Zheng et al. [32], who considered this zone a weak point in the concrete. Therefore, the mechanical behavior of concrete is strongly influenced by the properties of the cement-aggregate interface $[2,23,15,17,22,1]$. Using different methods and materials, these studies show that the concrete is strongly influenced by the volume fraction of the cementaggregate interface. The more the volume of the interface zone increases, the more the elastic modulus of concrete decreases. This phenomenon is even more emphasized when the water/cement ratio is higher [12]. Kawakami [9] and Husem [6] have investigated existing experimental relations between the compressive/bending strength of the concrete and the tensile/bending strength of the composite rock-cement paste/mortar. Their results show that the strength of concrete depends on that of the composite.

Some studies have been devoted to the direct characterization of the mechanical properties of the interface between aggregates and mortar or cement paste, by using tensile and shear tests. Thomas and Slate [31] found experimentally that the tensile strength of the interface varied, reaching 33-67\% of the tensile strength of cement paste or mortar. Nevertheless, these results vary with the nature of the aggregate as well as the shape and roughness of the surface. However, mesoscopic samples used for these studies did not provide the rupture localization at the interface. Rao and Prasad [5] and Guo et al. [5] found that the tensile strength of the interface between aggregate and mortar was about one third to half of the tensile strength of the mortar. Mielniczuk et al. [14] and Jebli et al. [7] have characterized the behavior of composites with spherical aggregate by direct compression and tensile tests. They showed that the proposed models on the macroscopic scale by Eurocode 2 to predict the behavior at rupture, are verified at the local scale considered here. In conclusion, all experimental studies to characterize the interface between aggregate and cement paste or mortar remain insufficient to access the interaction laws at this scale. The experimental studies presented in the literature to characterize the behavior of the cement-aggregate interface by tensile and shear tests have focused only on the strength. In the case of traction [31,24,5], no measurement of the deformation is available to deduce Young's modulus or the energy at rupture. A similar observation can be made about studies using shear tests [10,5]. Moreover, almost all studies are carried out at scales superior than that of the real aggregate in the concrete, and does not make it possible to take place in similar conditions at the scale of the aggregates in order to directly solicit the cement-aggregate interface.

In this context, the objective of this study is to determine the mechanical properties of the cement-aggregate interface in concrete by means of direct tensile and shear tests. To do that, innovative experimental devices were developed to directly load the cement-aggregate interface. After a presentation of materials and experimental devices, the results of direct tensile and shear tests are presented according to the hydration time. Then these results are analyzed and discussed.

\subsection{Experimental program}

In this study, composites and cement paste were used. To access the variables strength-strain, specific molds were manufactured to calibrate the dimensions of samples.

\subsubsection{Materials}

The selected cement is a Portland cement CEM II 32.5 CP2 NF EC used to prepare the cement paste with a Water/Cement ratio of 0.5 . Natural limestone parallelepipedic aggregates were made with dimensions of $10 \times 10 \times 15 \mathrm{~mm}^{3}$ (Fig. 1a).

The parallelepipedic aggregates were extracted from a single limestone rock as illustrated in Fig. 2. This rock is used for the manufacture of concrete aggregates. To ensure a similar surface roughness for all the aggregates, surface roughness saw-cuts were made in the same way for all aggregates. The limestone aggregates react with the cement paste, which leads to a good adhesion [8]. However, it does not prevent the formation of a cement-aggregate interface of about $25 \mu \mathrm{m}$ visible by Scanning Electron Microscopy (SEM) during 28 days of hardening (Fig. $1 \mathrm{~b}$ ). The material data is presented in Table 1.

\subsubsection{Geometry and sample preparation}

In this experimental study, two types of samples, whose dimensions are shown in Fig. 3, were made:

- For tensile tests, the samples are composites which have a thin cement paste layer of $2 \mathrm{~mm}$ between two limestone aggregates (Fig. 3a). This thin distance was selected because it approaches the Maximum Paste Thickness (MPT). The MPT depends on the volume fraction of the coarse and fine aggregates $g$, the maximum dry packing density of the coarse and fine aggregates mixture $g^{*}$ and the maximum aggregate diameter $d_{\max }[11]$ in the following relation:

$$
M P T=d_{\max }\left(\sqrt[3]{\frac{g^{*}}{g}}-1\right)
$$
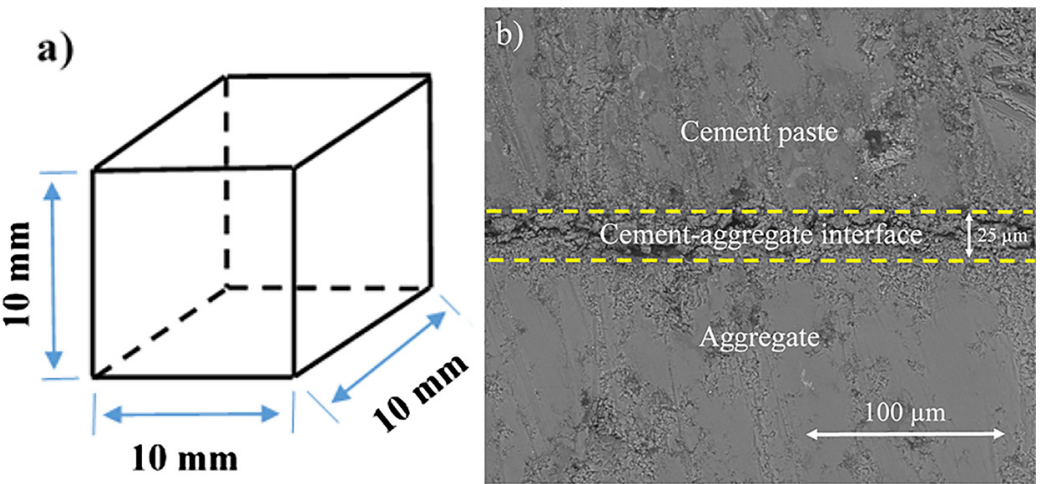

Fig. 1. a) Aggregate dimensions b) SEM Observation of cement paste-aggregate interphase at 28 days. 

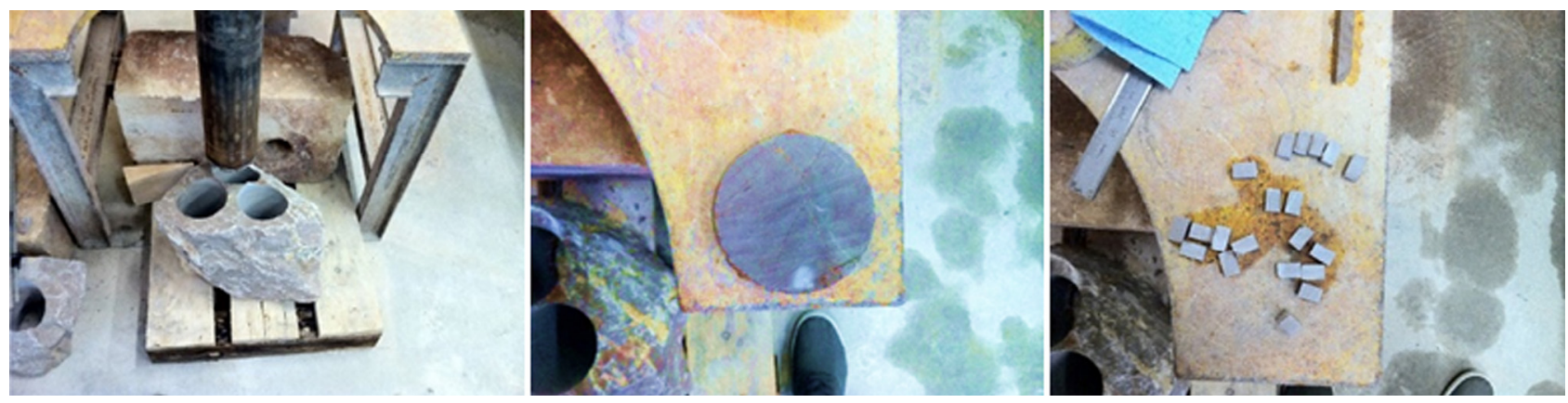

Fig. 2. The extraction steps of parallelepipedic aggregates.

Table 1

Material data of components used.

\begin{tabular}{lc}
\hline \multicolumn{2}{c}{ Aggregate } \\
\hline Type & Limestone \\
Density & $2.7 \mathrm{~g} / \mathrm{cm}^{3}$ \\
Dimension & $10 \times 10 \times 10 \mathrm{~mm}^{3}$ \\
& Cement paste \\
\hline Formula & CEM II/B-LL $32.5 \mathrm{~N}$ \\
Water/Cement ratio & 0.5 \\
Density & $2.2 \mathrm{~g} / \mathrm{cm}^{3}$ \\
\hline
\end{tabular}

On a macroscopic scale, the MPT is of the order of a few millimeters. In this study an MPT of about $2 \mathrm{~mm}$ is chosen.

- For direct shear tests, the samples are composites composed of a limestone aggregate and cement paste (Fig. 3b).

The composites were prepared using specific molds adapted to the desired dimensions. All samples are demolded after $24 \mathrm{~h}$ of hydration and are immediately immersed in water saturated with lime at a temperature of $21^{\circ} \mathrm{C}$ to prevent leaching and to decrease concrete shrinkage.

To obtain the mechanical properties of the cement paste, mechanical tests were also carried out on cement paste samples of the same dimensions as the composite shown in Fig. 3.

SEM tests on composites showed the presence of the cementaggregate interface with a thickness of $25 \mu \mathrm{m}$ (Fig. $1 \mathrm{~b}$ ). This thickness is close to that observed in a concrete with the same cement and the same aggregates $[33,18]$.

Concerning the effect of the shape of the aggregates, the plane surface considered increases the effect of the unilateral growth of the hydrates [3]. This phenomenon can lead to a more porous zone in the first 5 microns adjacent to the aggregates. On the other hand, the effect of micro-bleeding is less important in the composite than in the case of real concrete due to the mixing process [27].

\subsection{Methodology of experiments}

For each hydration time (2, 7, 15, 28 and 90 days), direct tensile and shear tests were carried out on three samples to measure strain and strength. All tests were performed on a "Mechanical Testing System (MTS)" by imposing a constant loading speed of $0.01 \mathrm{~mm} / \mathrm{s}$. Mechanical tests were recorded using a fast camera Vision Research Phantom V12 (acquisition speed of 10-40 thousands pictures per second), to measure the displacement during the tests by image correlation.

This technique was developed during the 1980s by Sutton et al. $[30,29]$, it is an alternative solution to the classic methods adopted for local field measurements of displacement or strain in structures, such as methods using strain gauges, LVDT displacement sensors or extensometers. The principle of this method is to determine the displacement and deformation fields of a solid. We do this by following the markings on the material at a reference time; by comparing two images taken at different loading steps, we follow the evolution. The equipment needed for using this technique are a high speed camera, a PC and a light source (Fig. 4a)).

Generally, a texture or a random pattern at the sample's surface, called mouchetis; is used. In our case this mouchetis is applied by spraying a white paint on the surface of the studied sample, previously tinged in black (Fig. 4b)). The two-dimensional kinematic measurements (displacement and deformation) are obtained through the software "Trait cin NRJ" developed in our laboratory. Time synchronization of various signals (kinematic and mechanical) is ensured by an electronic box "Synchrocam" which triggers all the acquisitions at the same time [4]. In our case, the displacement is calculated on a surface element near the rupture zone (a)

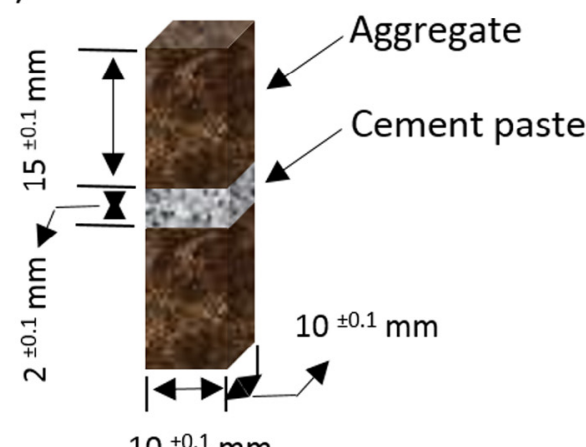

(b)

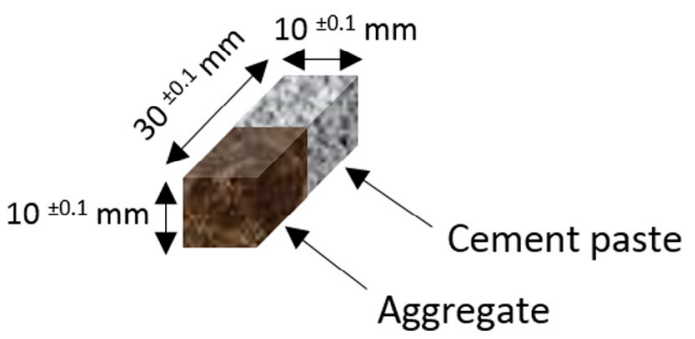

Fig. 3. Sample sizes for direct tensile test a) and direct shear test b). 
(a)

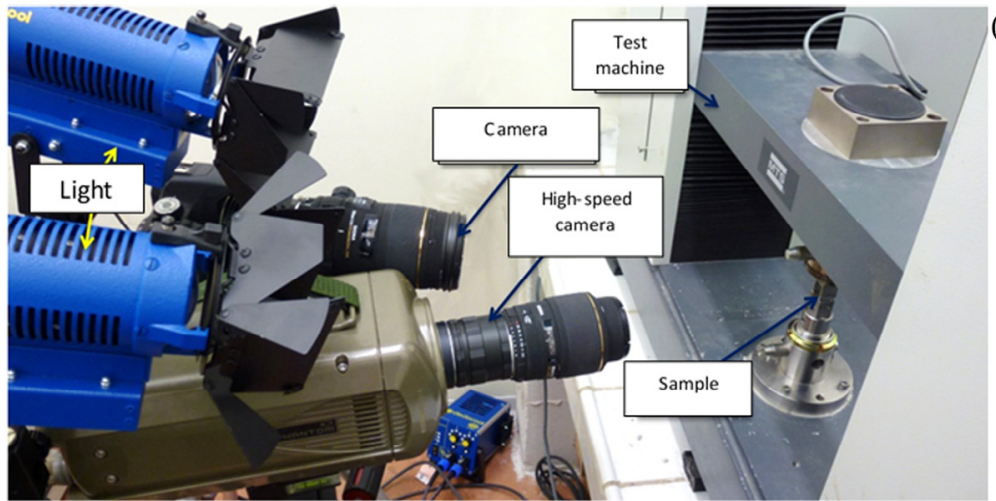

(b)

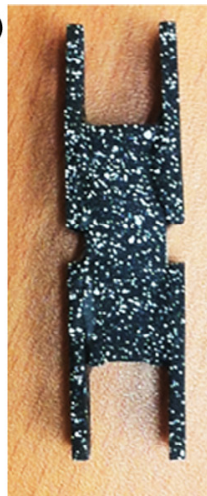

Fig. 4. Experimental devices for image correlation a) picture of sample with mouchetis b).
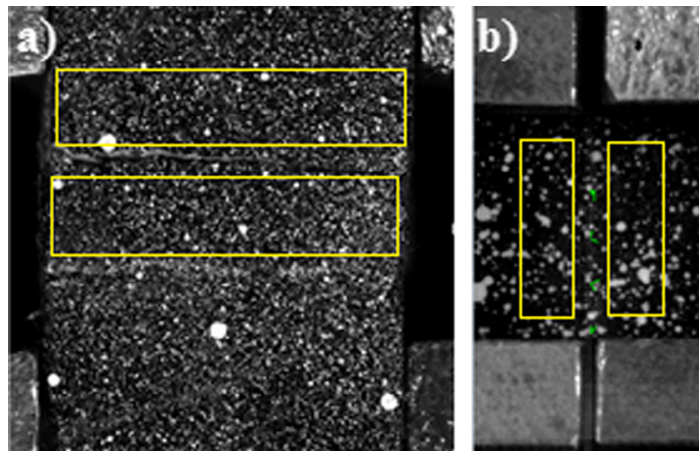

Fig. 5. Displacement calculation zones by image correlation for the direct tensile test a) and direct shear test $b$ ).

(Fig. 5), in order to calculate the jump displacement of the crack opening.

\subsubsection{Direct tensile test}

At a macroscopic scale, the direct tensile tests on concrete are difficult to achieve and often show widely dispersed results [25]. Several kinds of tensile tests are used to measure the tensile strength of the concrete, such as the splitting test or the threepoint bending test. The direct tensile test on the concrete at cement-aggregate interface scale, however, is less studied. To perform mechanical tests of direct tensile, experimental devices were designed and manufactured to be adapted to the size and shape of the tested samples. The principle of these tests is simple, but the preparation should be done carefully. Indeed, each misalignment, be it of the supports or in the tilt sample; induced parasitic bending which could change the results. Four metal shims
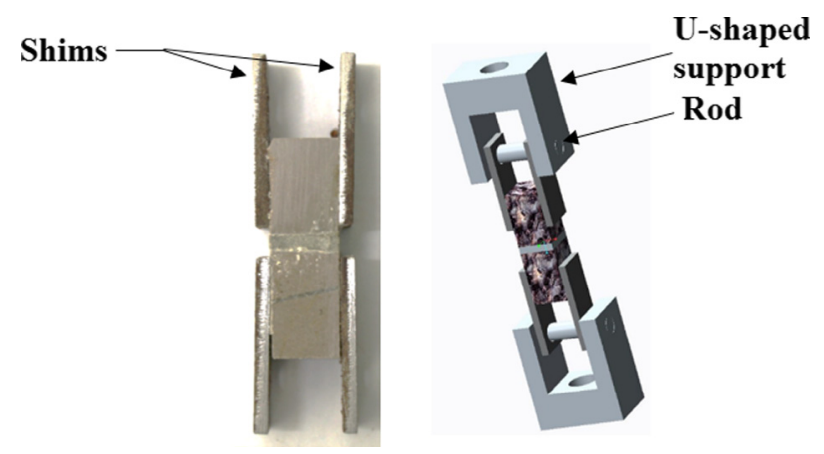

Fig. 6. Experimental device for direct tensile test. with holes are first fixed onto the sample as shown in Fig. 6. To perform the test, the sample is fixed with metal rods placed in the holes of the shims and of the "U" supports set on the testing machine.

\subsubsection{Direct shear test}

The direct shear test is widely used in soil mechanics to determine the macroscopic cohesion $c$ and the macroscopic internal friction angle $\Phi$. However, this test is one of the most difficult tests to be performed on cementitious materials, even more so when we want to characterize the cement-aggregate interface properties because of the lack of appropriate experimental tools. The objective of our original approach is to access to the Mohr-Coulomb law of cement-aggregate interface (local cohesion and local friction angle). To perform this test, experimental devices were designed and manufactured in such a way that the rupture is located at the cement-aggregate interface.

The device consists of two half-boxes, the distance between these two half-boxes being around $0.5 \mathrm{~mm}$ to minimize any bending near the cement-aggregate interface. Screws located at the rear of the half-boxes are used to fix the cement-aggregate interface in position in the middle of the device, thus avoiding movements of the sample during the test and allowing to apply a normal force. To measure this applied normal force, a force sensor (type LCMKD-200 N) is fixed at the rear of the sample. Fig. 7 illustrates the experimental device in which the sample is placed inside the two half-boxes.

The principle of the test is to apply a normal force $N$, to the cement-aggregate interface, monitored by the force sensor and inducing a confining stress $\sigma$. During the test, the displacement speed of one of the half-boxes is $0.01 \mathrm{~mm} / \mathrm{s}$, whereas the other remains still. During this displacement, the shear force $T$ is measured in the cement-aggregate interface, with a force sensor associated with the MTS machine, allowing access to the shear stress $\tau$. The relations between these variables are:
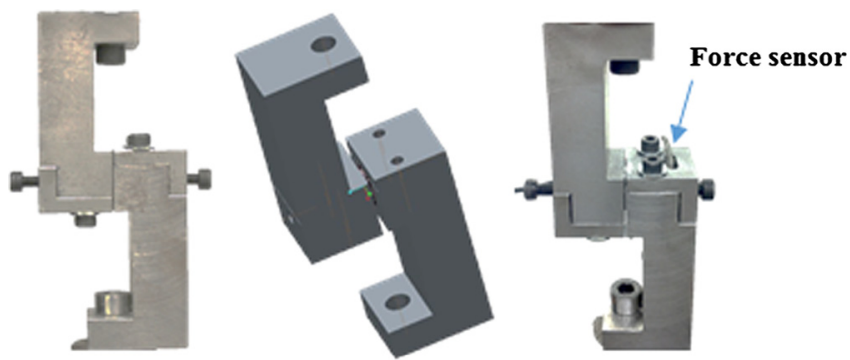

Fig. 7. Experimental device for direct shear test. 


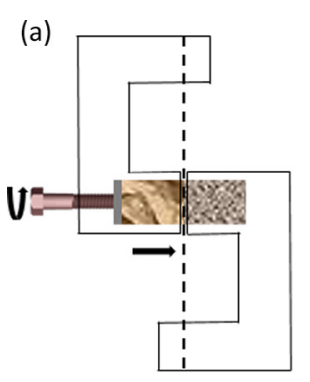

Align the supports and center the sample

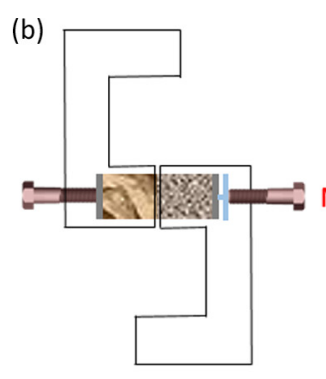

Position and fixe the force sensor

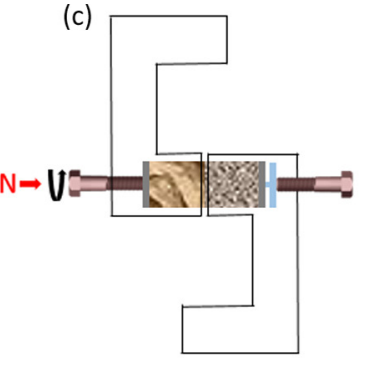

Apply the confining force

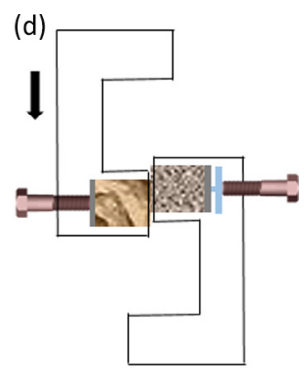

Shear the sample

Fig. 8. Principle of direct shear test.

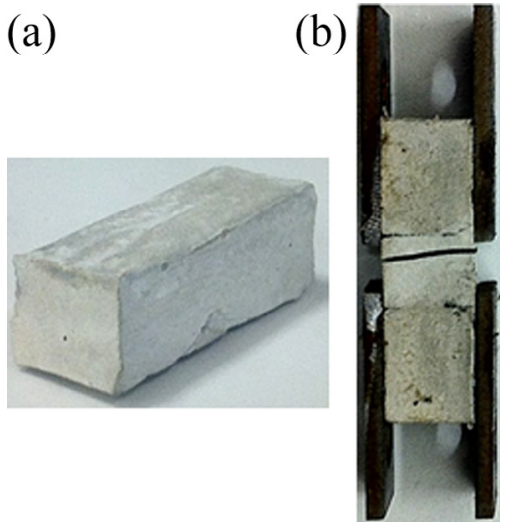

Fig. 9. Cement paste sample a) tensile test of cement paste b).

$\sigma=\frac{N}{A^{\prime}} \quad \tau=\frac{T}{A^{\prime}}$

where $A^{\prime}$ is the corrected section of the sample during the test. Fig. 8 shows the steps of the experimental procedure, from the introduction of the sample to the achievement of the direct shear test. For this test, strain corresponds to the sliding in the cementaggregate interface plane. The experimental device is designed in order to avoid any other degree of freedom.

By varying the normal force $\mathrm{N}$, the evolution of the shear strength $\tau_{r}$ in relation to the normal stress $\sigma$ applied during the test, can be deduced. For this study, two experimental protocols were carried out for direct shear tests:

- At different hydration times (2; 7; 15; 28 and 40 days) without normal stress $(\sigma=0 \mathrm{MPa})$,

- At 40 days hydration time for different normal stresses $(\sigma=0$; $0.5 ; 1$ and $1.5 \mathrm{MPa}$ ).

These two protocols allow obtaining the following information: for the first one, the evolution of the local cohesion $c$ at cementaggregate interface according to the hydration time and, for the latter, the local internal friction angle for a fixed hydration time. Mechanical tests were also carried out on cement paste using the same experimental devices of the composites as shown in Fig. 9.

\section{Experimental results}

Experimental results, obtained from the direct tensile and shear tests on cement paste and on the composite, are presented below.

\subsection{Direct tensile tests}

In this study, direct tensile tests are performed on samples constituted by two aggregates, binded by cement paste. The tests should be performed very carefully to avoid tilting the sample. In addition some other parameters could disturb the test results, such as microcracks at the cement-aggregate interface.

Fig. 10 shows the stress evolution as a function of the strain respectively for the composite "cement paste-aggregate"
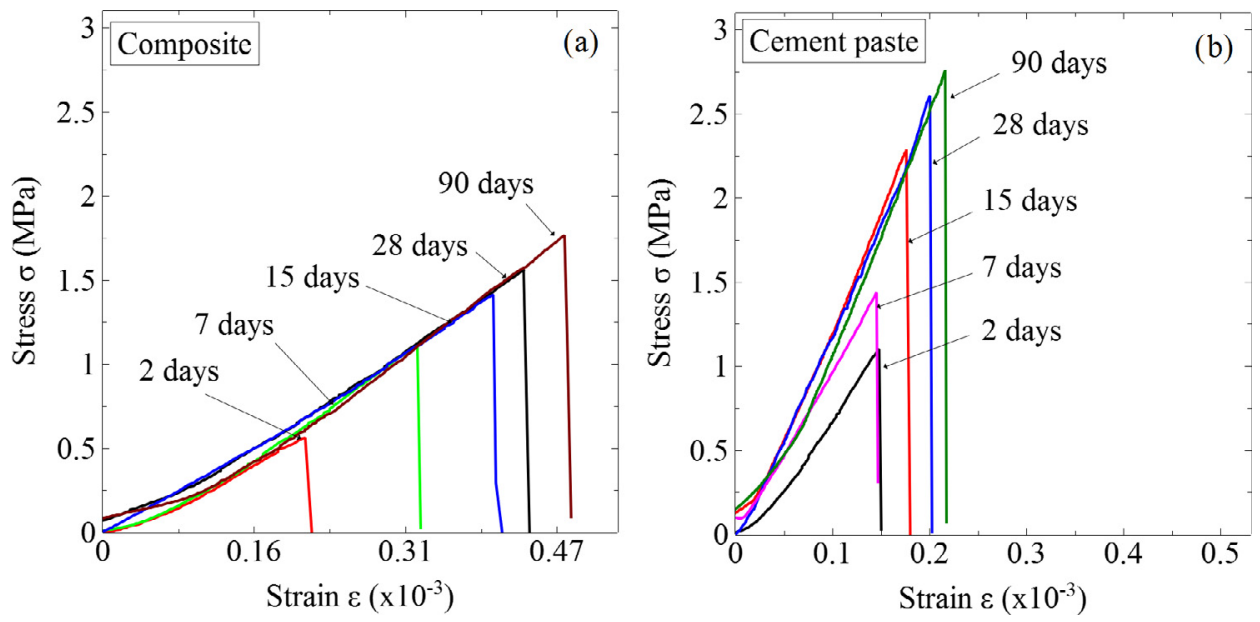

Fig. 10. Evolution of the stress vs strain during tensile tests for composite a) and cement paste b). 


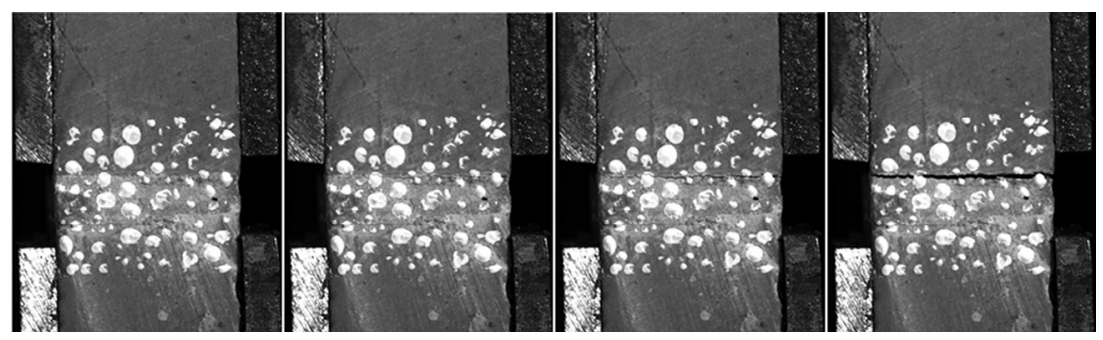

Fig. 11. Crack growth at cement-aggregate interface during tensile test.
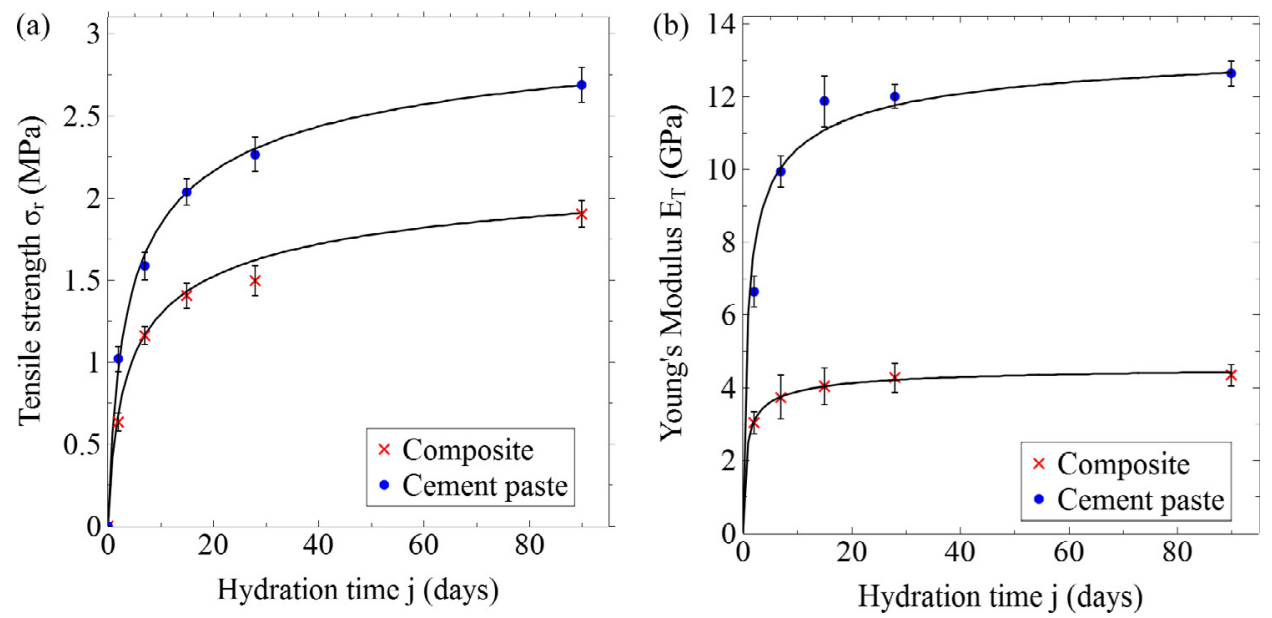

Fig. 12. Evolution of tensile strength a) and Young's modulus b) as a function of hydration time during tensile tests for composite and cement paste.

Table 2

Values of the modeling parameters for $\sigma_{r}(28), E_{T}(28)$ and $A$ of the composite and the cement paste.

\begin{tabular}{lll}
\hline & Composite & Cement paste \\
\hline$\sigma_{r}(28)(\mathrm{MPa})$ & 1.6 & 2.3 \\
$E_{T}(28)(\mathrm{GPa})$ & 4.3 & 12.0 \\
$A$ & 0.38 & 0.38 \\
\hline
\end{tabular}

(Fig. 10a) and for cement paste (Fig. 10b). This figures shows a significant increase in strength $\sigma_{r}(j)$ at the beginning of hydration, and a progressive stabilization after three weeks of hydration for the composite and cement paste.

For all the tensile tests, it is observed that all rupture on the composite occurs at the cement-aggregate interface (Fig. 11), which represents the weakest zone. Also a quasi-brittle behavior is observed for both samples. At this scale, there is a single crack that spreads quickly across the cement-aggregate interface, causing a sudden drop in the tensile stress at rupture. Similar results were obtained by Mielniczuk et al. [14] and Jebli et al. [7] using spherical aggregates. We also noted a gradual evolution in strain at break values over hydration time, it is more visible with the composite than with the cement paste.

Fig. 12 shows the evolution of the tensile strength $\sigma_{r}(j)$ (Fig. 12a) and tensile Young's modulus $E_{T}(j)$ (Fig. 12b) as functions of hydration time $j$ during tensile tests for composite and cement paste.

These evolutions were approximated by an exponential function, based on the equations proposed in Eurocode 2 [28] to estimate strength $\sigma_{r}(j)$ (eq. (3)) and the Young's modulus $E_{T}(j)$ (Eq. (4)) during the hydration time of composite and cement paste.

$\sigma_{r}(j)=\beta_{c c} \times \sigma_{r}(28)$
$E_{T}(j)=\left(\beta_{c c}\right)^{0.3} \times E_{T}(28)$

where $\sigma_{r}(28)$ is the strength of the samples corresponding to 28 days of hydration; $E_{T}(28)$ is the Young's modulus at 28 days and $\beta_{c c}$ is a coefficient which depends on the hydration time of the concrete according to the equation:

$\beta_{c c}=\exp \left[A\left(1-\left(\frac{28}{j}\right)^{1 / 2}\right)\right]$

where $A$ is a coefficient that depends on the type of cement.

Modellings proposed in Fig. 12 are given for the data presented in Table 2:

One can notice that the value of $A$ is the same for both the composite and the cement paste. Although more porous, the ITZ seems to harden at the same speed as the bulk paste. The experimental results show similar evolution of the strength and Young's modulus, but at each hydration time the characteristics of the cement paste are higher than those of the composite. In addition, ratio between composite and cement paste is about $2 / 3$ for the tensile strength and 1/3 for the Young's modulus.

These results tend to confirm that the cement paste-aggregate interface is the weakest zone in concrete. From the stress-strain curves (Fig. 10), the critical energy at rupture can generally be calculated using the following equation:

$W_{T}(j)=\int_{0}^{\epsilon_{r}} \sigma_{j} \mathrm{~d} \epsilon$

The linear behavior until the rupture occurs and the quasibrittle character of the rupture, allow us to approximate this energy as the triangle area under the stress - strain curve, in the following form: 


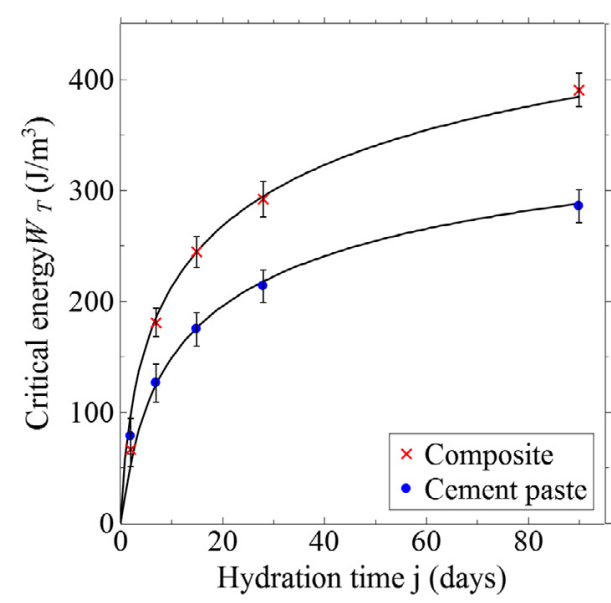

Fig. 13. Evolution of the critical energy for tensile test.

$W_{T}(j)=\frac{1}{2} \times \frac{\left(\sigma_{r}(j)\right)^{2}}{E_{T}(j)}$

where $\sigma_{r}(j)$ is the strength at $j$ days (Fig. 12a) and $E_{T}(j)$ is the tensile Young's modulus at $j$ days (Fig. 12b). Fig. 13 shows the evolution of the tensile critical energy $W_{T}(j)$ as a function of hydration time.

Fig. 13 shows the evolution of the energy at rupture and the Young's modulus as a function of the hydration time, there is an increase in energy with increasing hydration time until it gradually reaches a value of stability. The energy before rupture of the cement paste is lower compared to that of the interface. This is due to the higher strain at rupture for the composite, against a greater Young's modulus of the paste, about 3 times greater than that of the composite.

The difference of energy and the Young's modulus observed mean that the composite has a less brittle behavior than the cement paste. This observation can be explained by the high porosity in the cement-aggregate interface which gives a less brittle composite behavior.

\subsection{Direct shear tests}

The shear tests were performed on both the composite and the cement paste to study the mechanical behavior and define the shear laws of cement-aggregate interface and of cement paste, the latter as a reference to compare these characteristics with that of the cement-aggregate interface.

The curves of stress - strain, obtained during direct shear tests (without normal strength), are presented in Fig. 17 at different stages of hydration during the first 40 days for the cement paste and composite.

These figures show a fast increase in the shear strength and stiffness of the samples at the beginning of hydration. We noticed a decrease in the evolution speed of the shear strength after 28 days. In both the shear test and the tensile test, a quick drop in the stress is observed just after having reached its maximum. So an absence of the softening part of the stress-strain curve is observed in the macroscopic conventional mechanical behavior under shear stress. An increase of the strain values is also observed in the case of the composite with hydration, with slightly higher values for the composite beyond 7 days than for the cement paste. In this case the crack spreads quickly in the cement-aggregate interface. For the cement paste, breakings are conducted in the same strain value whatever the time of hydration.

The values of strength and energy at rupture $\tau_{r}(j)(\mathrm{MPa})$ and the tangential modulus $E_{s}(j)(\mathrm{GPa})$ as function of the hydration time $j$ (in days) for the shear test for both the composite and the cement paste, are shown on Fig. 15. The tangential modulus of the samples was estimated from the slope of the linear portion of the stressstrain curves (Fig. 14).

Fig. 15 shows a similar increase in strength and energy at rupture in shear test during hydration time for both types of samples. It is noted that the tangential modulus is about 1.5 times greater for the cement paste than for the composite. From the stress-strain curves of the shear tests (Fig. 14), the quasi-brittle behavior allows us to approximate the critical energy at rupture $W_{s}(j)$ as a function of hydration time in the form:

$W_{s}(j)=\frac{1}{2} \times \frac{\left(\tau_{r}(j)\right)^{2}}{E_{s}(j)}$

where $\tau_{r}(j)$ is the shear strength at rupture (Fig. 15a) and $E_{s}(j)$ is the tangential modulus (Fig. 15b) at $j$ days.

Fig. 16 shows an increase of the critical energy $W_{s}(j)$ with the hydration of samples. This time, contrary to the tensile test, the energy at rupture values is higher for the cement paste than for the composites.

After the characterization of the effect of hydration time on the shear strength; respectively for pure cement paste and the cement paste-aggregate interface, we focus on the influence of normal strength on the shear strengths. Direct shear tests are imposed to
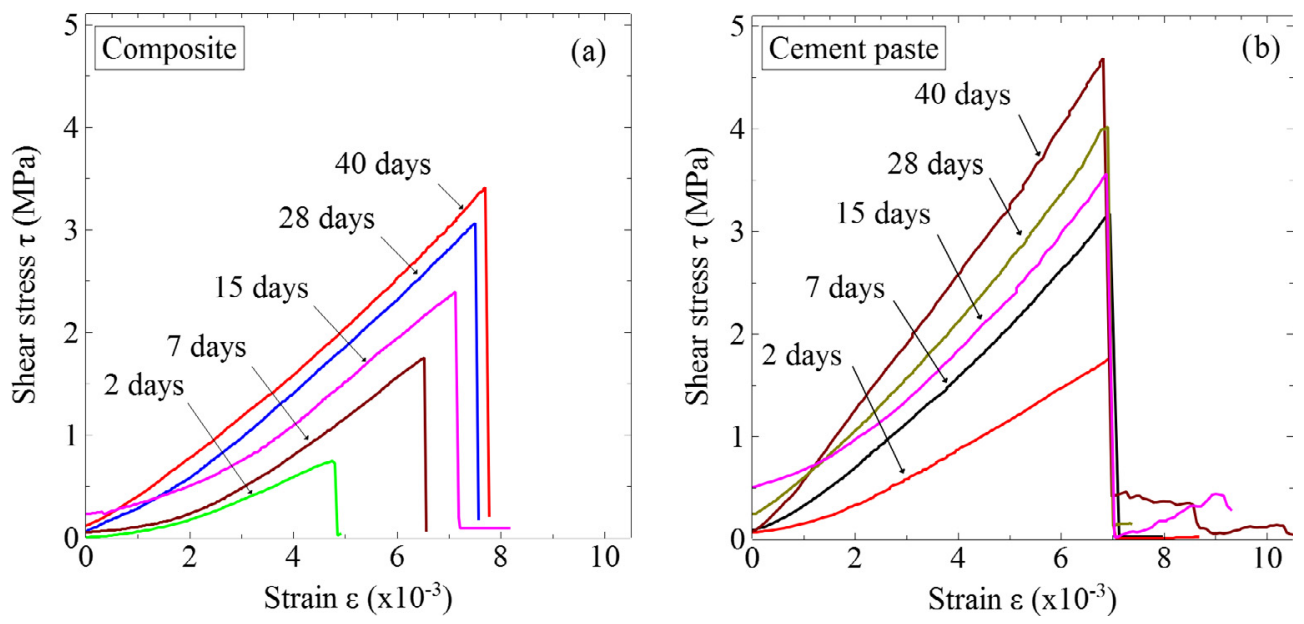

Fig. 14. Shear stress-strain curves during direct shear tests for composite a) and cement paste b). 

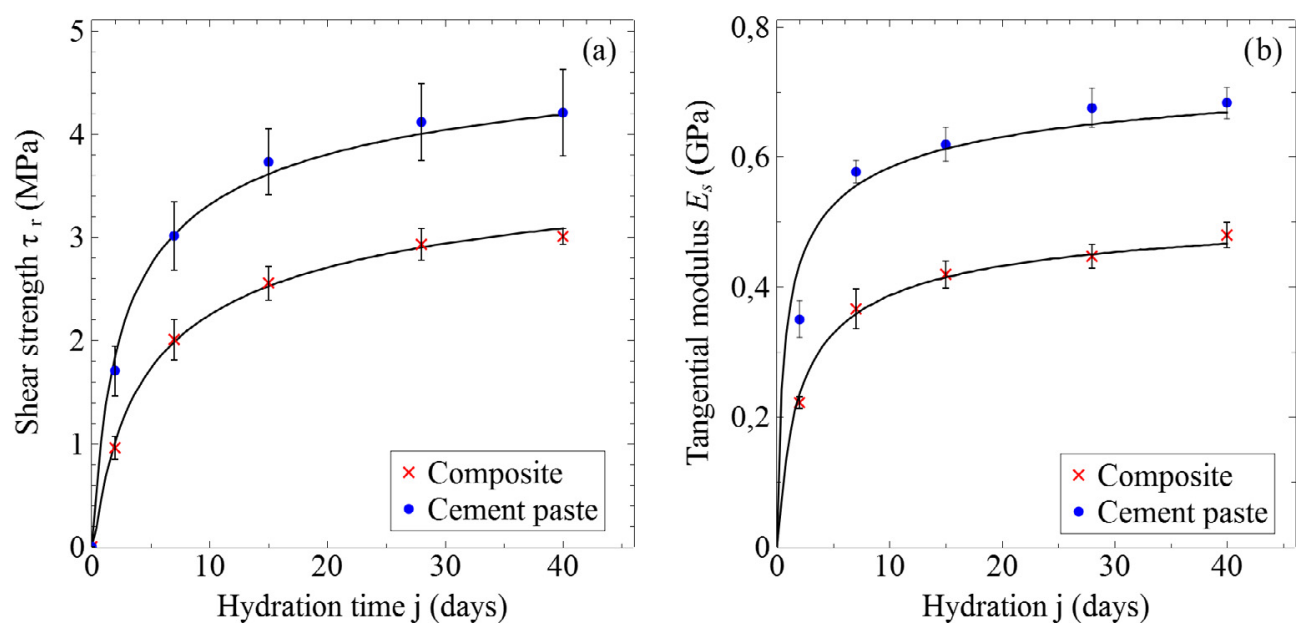

Fig. 15. Evolution of the local cohesion a) and the tangential modulus b) as a function of hydration time for the composite and the cement paste.

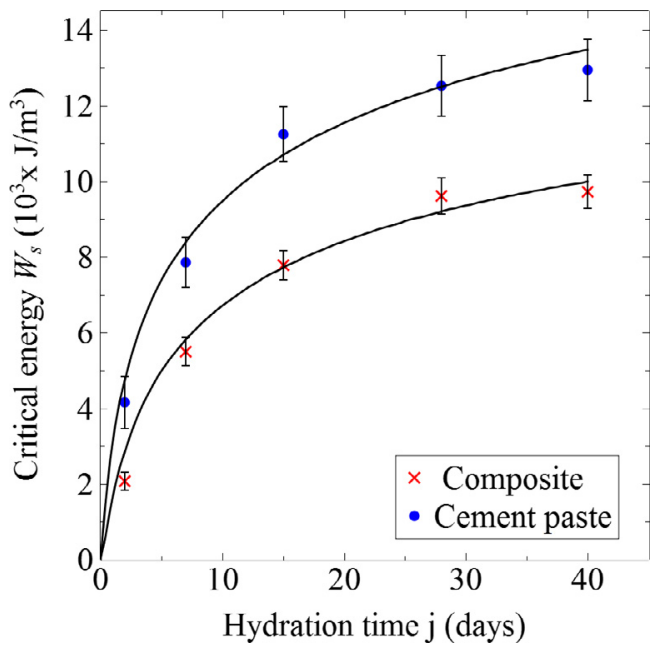

Fig. 16. Evolution of the energy at rupture as a function of hydration time for the composite and the cement paste.

a 40 days old sample, for different normal strengths. The samples, at a fixed hydration time (40 days), are subjected to direct shear tests at different normal strengths. The obtained strength-strain curves are shown in Fig. 17 for different confining strengths $(0$, $0.5,1,1.5 \mathrm{MPa}$ ). For all shear tests on the composite, we observe that the fracture is located at the cement-aggregate interface.

We noticed that there is a strong dependence of the mechanical behavior of composite and cement paste on normal stress. An increase in shear strength (peak value) with the application of normal stress for both samples is noted. The initial tangential modulus also increases in the same way. This evolution of shear strength according to normal strength (Fig. 18) is used to calculate the internal friction angle and cohesion according to Mohr-Coulomb criterion. We noticed a linear evolution of the shear strength with the increase of the normal stresses, for both cement paste and composite. According to Mohr-Coulomb the rupture criterion is defined by the following relationship:

$\tau_{r}(40)=\sigma \times \tan (\Phi)+c$

where $\tau_{r}(40)$ is the shear strength, $\sigma$ is the normal strength, $c$ is the ordinate of the failure envelope relative to the axis of $\tau$ and $\Phi$ is the failure envelope slope.

This evolution allows us to access the shear test law of the two types of samples. The evolution was obtained for (Table 3):

The results show that the local cohesion $c$ and local friction angle $\Phi$ of the paste are greater than those of the composite.
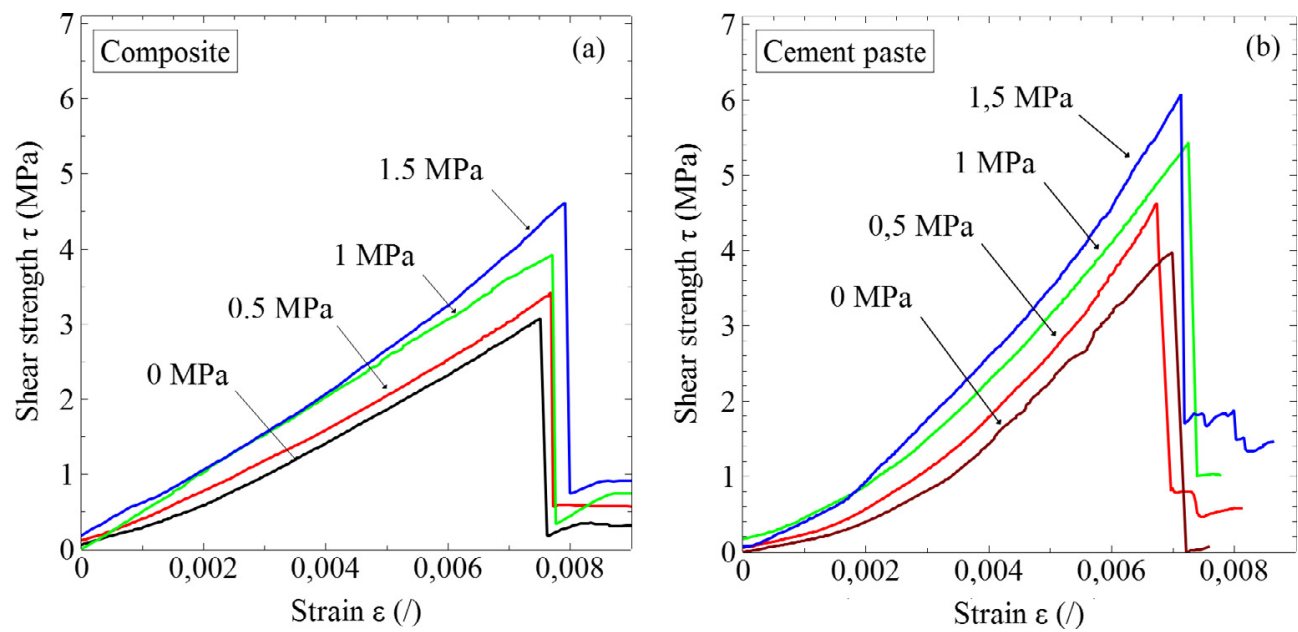

Fig. 17. stress-strain curves during direct shear tests for composite a) and cement paste b) for different normal stresses. 


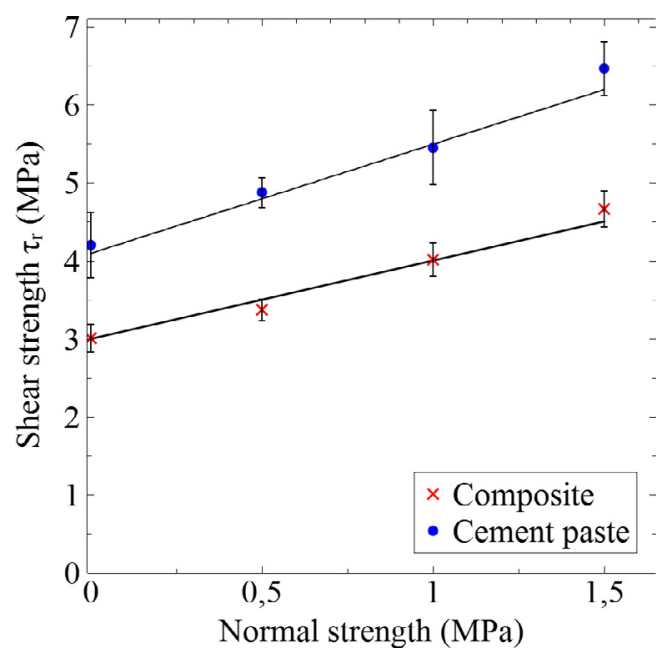

Fig. 18. Mohr - Coulomb straight line for the composite and the cement paste at 40 days.

Table 3

Local cohesion $c$ and local friction angle $\Phi$ of the composite and the cement paste at 40 days.

\begin{tabular}{lll}
\hline & Composite & Cement paste \\
\hline Local cohesion $c(\mathrm{MPa})$ & 3 & 4.1 \\
Local friction angle $\Phi\left(^{\circ}\right)$ & 47 & 54 \\
\hline
\end{tabular}

\section{Discussion}

Original tests at the scale of the cement paste - aggregate composite have been carried out to characterize the cement-aggregate interface properties. The results of the composite tensile tests showed that the tensile strength increases with hydration time, but with lower values than the cement paste. The composite tensile strength is almost $70 \%$ lower than that of cement paste. Thus the composite's Young's modulus is lower than that of the cement paste. A decrease of concrete Young's modulus with the increase of the volume fraction of cement-aggregate interface at macroscopic scale is also observed [2].

The calculation of tensile energy at rupture shows that the mechanical behavior of the composite is more less brittle than the cement paste due to the higher strain at rupture of the composite. This remark can be justified by the high porosity of the ITZ and the microstructure difference which affects the behavior of that zone. The evolution of these parameters is well approximated by an exponential function. This type of equation is commonly used to estimate the macroscopic strength of concrete. The evolution kinetics of the mechanical properties (strength and Young's modulus) is the same for the composite and the cement paste. These results of the tensile test at the scale of the cement-aggregates interface revealed that the mechanical properties of the ITZ are different than those of the cement paste.

Comparing the evolution of mechanical parameters during tensile tests on the scale of the ITZ with similar results for macroscopic samples, allow to conclude that the behavior of the samples is different. For macroscopic samples, a post peak of the strength-strain curve part can be distinguished, where the crack growth occurs [19].

An original experiment was conducted to study the mechanical behavior of the interphase by direct shear tests. The advantage of our experimental device is the possibility to apply different normal strengths, which provides access to the shear law on this scale. The results of the shear tests have shown that the local cohesion for the cement paste-aggregate interface and for the cement paste increases with the hydration time, but with high values of strength for the cement paste compared to those of the composite. The shear strength of the composite is lower than that of pure paste, but the strain of the composite is lightly higher. These results tend to confirm that the cement paste-aggregate interphase is the weakest zone in concrete. The application of the normal strengths increases the shear strength. This allowed the calculation of local cohesion and local friction angle of the cement-aggregate interface and the cement paste, in the same way as the Mohr-Coulomb on a macroscopic scale. Local cohesion and friction angle of cement paste are greater than those of the composite.

The results found at this scale show that the strengths of the cement-aggregate interface are low compared to those of the cement matrix. For both tests the strain values of the composite are higher than those of cement paste. Indeed, the less brittle character of the composite than of the cement paste was stronger marked for the tensile test than for the shear test. The difference of the strengths can be explained by the microstructure and the high porosity of the interface compared to that of the cement paste. These differences influence the properties and the mechanical behavior of the ITZ. Thus, it is not surprising to find unusual and different strength-strain curves compared to those observed at the macroscopic scale and many other tests with good repeatability. At the macroscopic scale, the usual laws not only take into account local mechanisms up-scaled at the macroscopic level but also a statistical disorder which adds another complexity.

These experimental results on the resistance, Young's modulus and the energy at rupture are expected by the scientific community interested in the change of scales in the study of the mechanical behavior of concrete. Indeed, these quantities, which are not available, are most overestimated in numerical simulations based on the Cohesive Zone Model (CZM) [13,21]. The strategy of these models consists in transposing the local behavior on the macroscopic scale (VER) in order to deduce an equivalent behavior.

\section{Conclusion}

An original experiment was performed to study the tensile strength and shear strength of a cement-aggregate interface and a cement paste. The ITZ is thin and has a higher porosity than the cement paste. This specificity significantly affects the mechanical properties of concrete on the initiation and propagation of the crack in the least resistant zones in concrete. The experimental results showed that the cement-aggregate interface is the weakest zone in concrete. The tensile test showed that the composite has a less brittle character than cement paste.

These preliminary results are a first approach to characterize the mechanical properties of the interface with the original experimental tools. These tools, developed at local scale, will provide support in understanding more global phenomena at a macroscopic scale. Indeed, modeling the mechanical behavior at the scale of cement-aggregate interface requires significant experimental investigations to complement computer codes data using the discrete element method. Among these investigations, it is necessary to consider the intrinsic material properties such as the type of aggregates and their surface states (shape, roughness, porosity, etc.), and the type of mortar and its composition (mineralogy, water/cement ratio, sand/cement ratio, additives, etc.).

\section{Acknowledgements}

We acknowledge Bertrand Wattrisse and Tarik Madani for their assistance to use Trait Cin NRJ software, and Gille Camp for his help in the manufacturing of experimental devices. 


\section{References}

[1] T. Akçaoğlu, M. Tokyay, T. Çelik, Effect of coarse aggregate size and matrix quality on itz and failure behavior of concrete under uniaxial compression, Cem. Concr. Compos. 26 (2004) 633-638.

[2] K. Alexander, J. Taplin, Analysis of the strength and fracture of concrete based on an unusual insensitivity of cement-aggregate bond to curing temperature, Aust. J. Appl. Sci. 15 (1964) 160-170.

[3] D.P. Bentz, E.J. Garboczi, Digitized simulation model for microstructural development, Ceram. Trans. 211 (1991) 16.

[4] A. Chrysochoos, B. Wattrisse, J.-M. Muracciole, Y. El Kaïm, Fields of stored energy associated with localized necking of steel, J. Mech. Mater. Struct. 4 (2009) 245-262.

[5] X. Gu, L. Hong, Z. Wang, F. Lin, Experimental study and application of mechanical properties for the interface between cobblestone aggregate and mortar in concrete, Constr. Build. Mater. 46 (2013) 156-166.

[6] M. Husem, The effects of bond strengths between lightweight and ordinary aggregate-mortar, aggregate-cement paste on the mechanical properties of concrete, Mater. Sci Eng. A 363 (2003) 152-158.

[7] M. Jebli, F. Jamin, E. Garcia-Diaz, M. El Omari, M.S. El Youssoufi, Influence of leaching on the local mechanical properties of an aggregate-cement paste composite, Cem. Concr. Compos. 12 (2016) 241-250.

[8] S. Kamali-Bernard, D. Keinde, F. Bernard, Effect of aggregate type on the concrete matrix/aggregates interface and its influence on the overall mechanical behavior. a numerical study, Key Eng. Mater. 617 (2014) 14-17.

[9] H. Kawakami, Effect of aggregate type on the mechanical behaviour of concrete, in: International union of testing and research laboratories for materials and construction, Toulouse (1992) pp. 179-186.

[10] Y. Kosaka, Y. Tanigawa, M. Kawakami, Effect of coarse aggregate on fracture of concrete (part 1), J. AIJ 228 (1975) 1-11.

[11] F.D. Larrard, Concrete Mixture Proportioning. E \& FN Spon, (1999).

[12] K. Lee, J. Park, A numerical model for elastic modulus of concrete considering interfacial transition zone, Cem. Concr. Res. 38 (2008) 396-402.

[13] E. Malachanne, M. Salgues, M. Jebli, F. Jamin, A cohesive zone model for the characterisation of the interfacial transition zone (its) between cement paste and aggregates, in: ECCOMAS Congress 2016.

[14] B. Mielniczuk, M. Jebli, F. Jamin, M.S. El Youssoufi, C. Pelissou, Y. Monerie, Characterization of behavior and cracking of a cement paste confined between spherical aggregate particles, Cem. Concr. Res. 79 (2016) 235-242.

[15] S. Mindess, Tests to determine the mechanical properties of the interfacial zone. RILEM report, (1996) pp. 47-63.

[16] S. Mindess, J.F. Young, D. Darwin, Concrete, Prentice Hall, 2003.
[17] A.R. Mohamed, W. Hansen, Micromechanical modeling of crack-aggregate interaction in concrete materials, Cem. Concr. Compos. 21 (1999) 349-359.

[18] P.J.M. Monteiro, P.K. Mehta, Improvement of the aggregate-cement paste transition zone by grain refinement of hydration products, in: Proceedings of the 8th international congress on the chemistry of cement, Rio de janeiro (pp. 433-437). vol. 2 (1986).

[19] I. Odler, M. Röler, Investigations on the relationship between porosity, structure and strength of hydrated portland cement pastes. ii. effect of pore structure and of degree of hydration, Cem. Concr. Res. 15 (1985) 401-410.

[20] J.P. Ollivier, Contribution à l'étude de l'hydratation de la pâte de ciment Portland au voisinage des granulats Ph.D. thesis, Université Paul Sabatier, Toulouse, 1981.

[21] F. Perales, S. Bourgeois, A. Chrysochoos, Y. Monerie, Two field multibody method for periodic homogenization in fracture mechanics of nonlinear heterogeneous materials, Eng. Fract. Mech. 75 (2008) 3378-3398.

[22] G. Prokopski, J. Halbiniak, Interfacial transition zone in cementitious materials, Cem. Concr. Res. 30 (2000) 579-583.

[23] G. Ramesh, E. Sotelino, W. Chen, Effect of transition zone on elastic moduli of concrete materials, Cem. Concr. Res. 26 (1996) 611-622.

[24] G.A. Rao, B.R. Prasad, Influence of the roughness of aggregate surface on the interface bond strength, Cem. Concr. Res. 32 (2002) 253-257.

[25] M. Saito, M. Kawamura, Resistance of the cement-aggregate interfacial zone to the propagation of cracks, Cem. Concr. Res. 16 (1986) 653-661.

[26] K. Scrivener, Characterisation of the itz and its quantification by test methods. RILEM report, (1999) pp. 3-18.

[27] K.L. Scrivener, K.M. Nemati, The percolation of pore space in the cement paste/ aggregate interfacial zone of concrete, Cem. Concr. Res. 26 (1996) 35-40.

[28] B. Standards, Eurocode 2: Design of Concrete Structures: Part 1-1: General Rules and Rules for Buildings, British Standards Institution, 2004.

[29] M. Sutton, C. Mingqi, W. Peters, Y. Chao, S. McNeill, Application of an optimized digital correlation method to planar deformation analysis, Image Vis. Comput. 4 (1986) 143-150.

[30] M. Sutton, W. Wolters, W. Peters, W. Ranson, S. McNeill, Determination of displacements using an improved digital correlation method, Image Vision Comput. 1 (1983) 133-139.

[31] T. Thomas, F.O. Slate, Tensile bond strength between aggregate and cement paste or mortar, In: Journal Proceedings (pp. 465-486). vol. 60 (1963).

[32] J. Zheng, C. Li, X. Zhou, Thickness of interfacial transition zone and cement content profiles around aggregates, Mag. Concr. Res. 57 (2005) 397-406.

[33] R. Zimbelmann, A contribution to the problem of cement-aggregate bond, Cem. Concr. Res. 15 (1985) 801-808. 